\title{
Update of a multiphase transport model with modern parton distribution functions and nuclear shadowing
}

\author{
Chao Zhang, ${ }^{1}$ Liang Zheng, ${ }^{2,1}$ Feng Liu, ${ }^{1}$ Shusu Shi, ${ }^{1,{ }^{*}}$ and Zi-Wei Lin ${ }^{1,3, \dagger}$ \\ ${ }^{1}$ Key Laboratory of Quark \& Lepton Physics (MOE) and Institute of Particle Physics, Central China Normal University, Wuhan 430079, China \\ ${ }^{2}$ China University of Geosciences, Wuhan 430074, China \\ ${ }^{3}$ Department of Physics, East Carolina University, Greenville, North Carolina 27858, USA
}

(Received 12 March 2019; published 24 June 2019)

\begin{abstract}
A multiphase transport (AMPT) model has been successful in explaining a wide range of observables in relativistic heavy ion collisions. In this work, we implement a newer set of free proton parton distribution functions and an impact parameter-dependent nuclear shadowing in the AMPT model. After refitting the parameters of the two-component initial condition model to the experimental data on $p p$ and $p \bar{p}$ total and inelastic cross sections from $\sqrt{s} \sim 4 \mathrm{GeV}$ to $13 \mathrm{TeV}$, we study particle productions in $p p$ and $A A$ collisions. We show that the updated AMPT model with string melting can reasonably describe the overall particle yields and transverse momentum spectra for both $p p$ and $A A$ collisions at RHIC and LHC energies after we introduce a nuclear scaling of the minijet transverse momentum cutoff for $A A$ collisions at LHC energies that is motivated by the color glass condensate. Since heavy flavor and high- $p_{\mathrm{T}}$ particles are produced by perturbative-QCD processes and thus directly depend on parton distribution functions of nuclei, the updated AMPT model is expected to provide a more reliable description of these observables.
\end{abstract}

DOI: 10.1103/PhysRevC.99.064906

\section{INTRODUCTION}

Experimental results from the Relativistic Heavy Ion Collider (RHIC) and the Large Hadron Collider (LHC) [1-3] indicate that a hot and dense medium with partonic degrees of freedom, namely, the quark-gluon plasma (QGP), is created in heavy ion collisions at high energies. To study the properties of QGP, various theoretical methods and models are being developed including a multiphase transport (AMPT) model [4]. The AMPT model aims to describe the whole phase space evolution of heavy-ion collisions as it contains four main components: the fluctuating initial condition, partonic interactions, hadronization, and hadronic interactions. The AMPT model has been widely used to simulate the evolution of the dense matter created in high energy heavy ion collisions. In particular, the string melting version of the AMPT model $[4,5]$, which converts the created matter in the overlap volume into parton degrees of freedom, can well describe the anisotropic flows and particle correlations in collisions of small or large systems at both RHIC and LHC energies [4-8].

However, the current public AMPT model (up to version v1.26/v2.26 [9]) uses the old Duke-Owens parton distribution functions for the free proton and a schematic nuclear shadowing parametrization from the HIJING 1.0 model [10,11]. Therefore, it significantly underestimates the gluon and quark distributions at small $x$. This would lead to significant uncertainties in its predictions on heavy flavor and/or high- $p_{\mathrm{T}}$ observables, because those particles are initially produced by

\footnotetext{
*shiss@mail.ccnu.edu.cn

†linz@ecu.edu
}

perturbative-QCD processes and thus directly depend on the parton distribution functions (PDFs) of nuclei. To improve the AMPT model for high energy nuclear collisions, especially on heavy flavor and high- $p_{\mathrm{T}}$ observables, we incorporate in this study a newer set of free proton parton distribution functions (the CTEQ6.1M set [12]) and an impact parameter-dependent EPS09sNLO nuclear shadowing [13] in an updated AMPT model.

The paper is organized as follows. After the introduction, we describe the initial condition of the AMPT model in Sec. II, including the HIJING two-component model, the CTEQ6.1M (NLO) parton distribution functions for the free proton, the impact parameter-dependent EPS09s nuclear shadowing functions, and our determination of the energy dependence of two key parameters $\left(p_{0}\right.$ and $\left.\sigma_{\text {soft }}\right)$ in the twocomponent model. We then investigate particle rapidity distributions and transverse momentum spectra from the string melting version of the updated AMPT model in Sec. III, including our results for both $p p$ collisions and $A A$ collisions at RHIC and LHC energies in comparison with the experimental data. More discussions can be found in Sec. IV including the effects of nuclear shadowing and the nuclear scaling of the minijet transverse momentum cutoff $p_{0}$ on particle productions in $A A$ collisions. Finally, a summary is given in Sec. V.

\section{THE INITIAL CONDITION OF THE AMPT MODEL}

The string melting version of a multiphase transport model [4,5] contains four main parts: the fluctuating initial conditions based on the HIJING two-component model [10,11], 
elastic parton scatterings modeled by the ZPC parton cascade [14], a spatial quark coalescence model to describe the hadronization of the parton matter [5,7], and a hadron cascade based on the ART model $[4,15]$. When we incorporate new parton distribution functions of nuclei in the AMPT model, two key parameters in the HIJING two-component model, $p_{0}$ and $\sigma_{\text {soft }}$, need to be retuned to describe the cross sections of $p p$ and $p \bar{p}$ collisions.

\section{A. The HIJING two-component model}

The HIJING model [10,11], which combines jet production that scales with the number of binary collisions with string fragmentation, provides the initial condition of heavy ion collisions in the AMPT model. In the HIJING model, the primary interactions between the projectile and target are divided into soft and hard components with a transverse momentum scale $p_{0}$. An interaction with a momentum transfer larger than $p_{0}$ is considered to be a hard process and its production is calculated with perturbative QCD. However, the soft component with a momentum transfer below $p_{0}$ is considered to be nonperturbative and characterized by the cross section $\sigma_{\text {soft }}$.

The inclusive jet differential cross section [16] in HIJING is determined by

$$
\frac{d \sigma_{\text {jet }}}{d p_{\mathrm{T}}^{2} d y_{1} d y_{2}}=K \sum_{a, b} x_{1} f_{a}\left(x_{1}, Q^{2}\right) x_{2} f_{b}\left(x_{2}, Q^{2}\right) \frac{d \sigma^{a b}}{d \hat{t}},
$$

where $p_{\mathrm{T}}$ is the transverse momentum transfer, $y_{1}$ and $y_{2}$ are, respectively, the rapidity of the two produced partons, $x_{1}$ and $x_{2}$ are, respectively, the fraction of the momentum carried by the two initial partons, $f_{a}\left(x_{1}, Q^{2}\right)$ is the parton distribution function of parton type $a$ at the $x$ value of $x_{1}$ and factorization scale $Q^{2}$, and $\sigma^{a b}$ is the cross section between parton types $a$ and $b$. Note that the $K$ factor in Eq. (1) aims to account for higher-order corrections, since the minijet cross section is calculated at the leading order (even though the PDFs including nPDFs are evaluated at NLO), and the $K$ factor takes the same value (2.5) as in the HIJING1.0 model since version 1.33 and the previous AMPT model for minijet productions. The total inclusive jet cross section (for the production of minijet gluons and $u / d / s$ quarks) is then obtained by integrating the above with a transverse momentum cutoff $p_{0}[10]$ :

$$
\sigma_{\text {jet }}(s)=\frac{1}{2} \int_{p_{0}^{2}}^{s / 4} d p_{\mathrm{T}}^{2} d y_{1} d y_{2} \frac{d \sigma_{\text {jet }}}{d p_{\mathrm{T}}^{2} d y_{1} d y_{2}} .
$$

By introducing a soft interaction cross section $\sigma_{\text {soft }}$, one can write an eikonal function $[17,18]$ as

$$
\chi(b, s)=\frac{1}{2} \sigma_{\text {soft }}(s) T_{N}(b, s)+\frac{1}{2} \sigma_{\text {jet }}(s) T_{N}(b, s),
$$

where $T_{N}(b, s)$ is the partonic overlap function between two nucleons at impact parameter $b[10,11]$. Then in the eikonal formalism, the total, elastic, and inelastic cross section of the nucleon-nucleon collisions can be written, respectively, as

$$
\sigma_{\text {tot }}=2 \pi \int_{0}^{\infty} d b^{2}\left[1-e^{-\chi(b, s)}\right]
$$

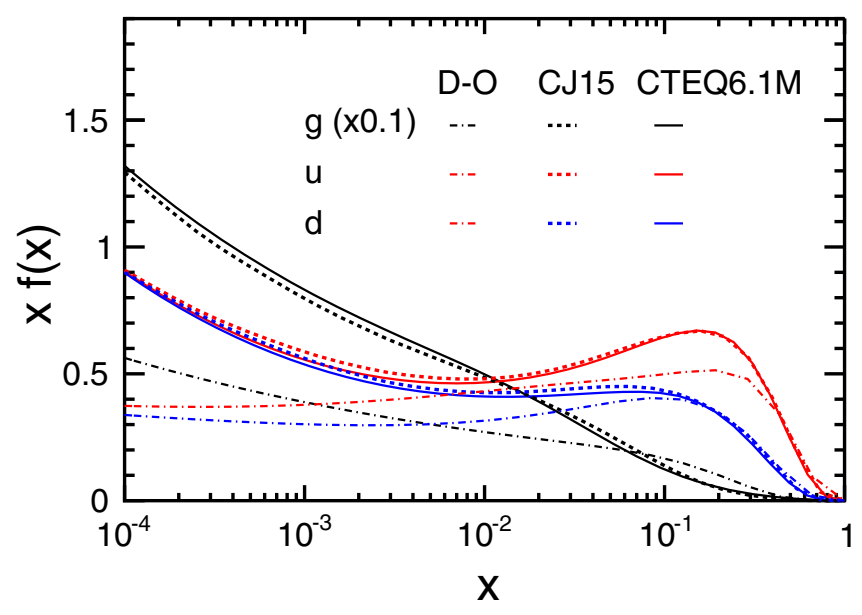

FIG. 1. Parton density distributions of the free proton (at $Q^{2}=$ $10 \mathrm{GeV}^{2}$ ) from the CTEQ6.1M set (solid curves) in comparison with the old Duke-Owens set (dot-dashed) and the more recent CJ15 set (dashed).

$$
\begin{aligned}
& \sigma_{\mathrm{el}}=\pi \int_{0}^{\infty} d b^{2}\left[1-e^{-\chi(b, s)}\right]^{2}, \\
& \sigma_{\text {in }}=\pi \int_{0}^{\infty} d b^{2}\left[1-e^{-2 \chi(b, s)}\right],
\end{aligned}
$$

and they depend on both $p_{0}$ and $\sigma_{\text {soft }}$.

\section{B. Parton distribution functions of the free proton}

The HIJING 1.0 model in the current AMPT model uses the Duke-Owens parton distribution function set 1 [19] for the free proton. However, it is well known that the Duke-Owens PDFs are outdated, especially when the minijet productions reach the small- $x$ region of the parton distributions at high energies [20]. So in this work we implement the newer CTEQ6.1M set [12] for the parton PDFs of free proton (and free neutron). A similar update of parton PDFs has been done for the HIJING model, where the GRV PDFs [21] were used in the updated HIJING 2.0 model [22] to replace the Duke-Owens PDFs.

Figure 1 compares the parton density distributions (PDFs multiplied by $x$ ) from the Duke-Owens, CTEQ6.1M, and CJ15 sets for the gluon, u-quark, and d-quark. Note that the gluon distributions have been scaled down by a factor of ten. We see that all three distributions in the CTEQ6.1M parametrization are quite different from the old Duke-Owens set and are much higher at small $x$ values. In addition, differences between the CTEQ6.1M PDFs and the more recent CJ15 PDFs [23] are quite small.

\section{Parton distribution functions in a nucleus}

Nuclear shadowing functions describe the modifications of parton distribution functions in a nucleus relative to a simple superposition of parton distribution functions in the nucleon. Since we will be interested in describing nucleusnucleus collisions at various impact parameters, we implement the impact parameter-dependent EPS09sNLO nuclear 


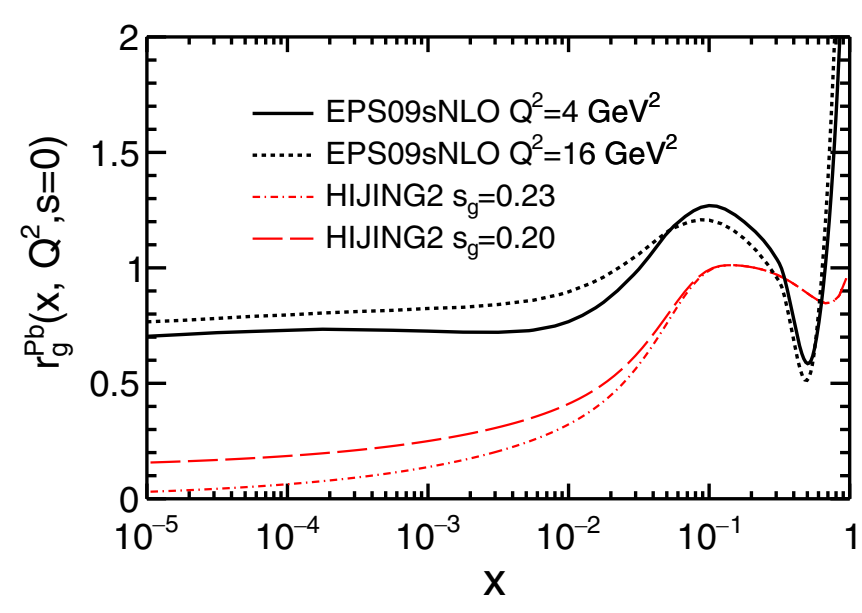

FIG. 2. Comparison of the nuclear shadowing functions of gluons at the center of a lead nucleus from the EPS09s NLO set at two different $Q^{2}$ values and from the HIJING 2 parametrization with two different values for the $s_{g}$ parameter.

shadowing functions [13] instead of the spatially independent (averaged) EPS09 nuclear shadowing [24]. They describe the spatial dependence of nuclear PDFs (nPDFs) and are based on data from deep inelastic lepton-nucleus scatterings, DrellYan dilepton productions, and specifically pion productions measured at RHIC [25] which improve the determination of the gluon densities. Note that the EPS09sNLO set was calculated with the CTEQ6M set as the free proton PDFs, which is almost equivalent in every respect to the CTEQ6.1M set [26].

For an average bound proton in a nucleus, the distribution function of parton flavor $i$ can be written as

$$
f_{i}^{p / A}\left(x, Q^{2}\right) \equiv R_{i}^{A}\left(x, Q^{2}\right) f_{i}^{p}\left(x, Q^{2}\right),
$$

where $f_{i}^{p}\left(x, Q^{2}\right)$ is the corresponding PDF in the free proton. Here $R_{i}^{A}\left(x, Q^{2}\right)$ represents the spatially-averaged nuclear modification or shadowing function, which mainly contains three effects depending on the $x$ range: the shadowing effect, anti-shadowing effect, and the EMC effect. It is an integral of the spatially dependent nuclear shadowing function as given by

$$
R_{i}^{A}\left(x, Q^{2}\right) \equiv \frac{1}{A} \int d^{2} \mathbf{s} T_{A}(\mathbf{s}) r_{i}^{A}\left(x, Q^{2}, \mathbf{s}\right)
$$

In the above, $T_{A}(\mathbf{s})$ is the nuclear thickness function at transverse position $\mathbf{s}$, and $r_{i}^{A}\left(x, Q^{2}, \mathbf{s}\right)$ is the spatially dependent nuclear shadowing.

Figure 2 shows the gluon shadowing functions at the center of a lead nucleus from the EPS09s NLO set at two different $Q^{2}$ values and from the HIJING 2.0 model at two different $s_{g}$ values suggested for LHC energies [22]. We see that the EPS09sNLO gluon shadowing at small $x$ is much weaker than the HIJING shadowing. Note that the current AMPT model uses the HIJING 1.0 nuclear shadowing parametrization, which is spatially dependent but independent of $Q^{2}$ or the parton flavor $[4,10]$ and similar to the HIJING 2.0 nuclear shadowing.

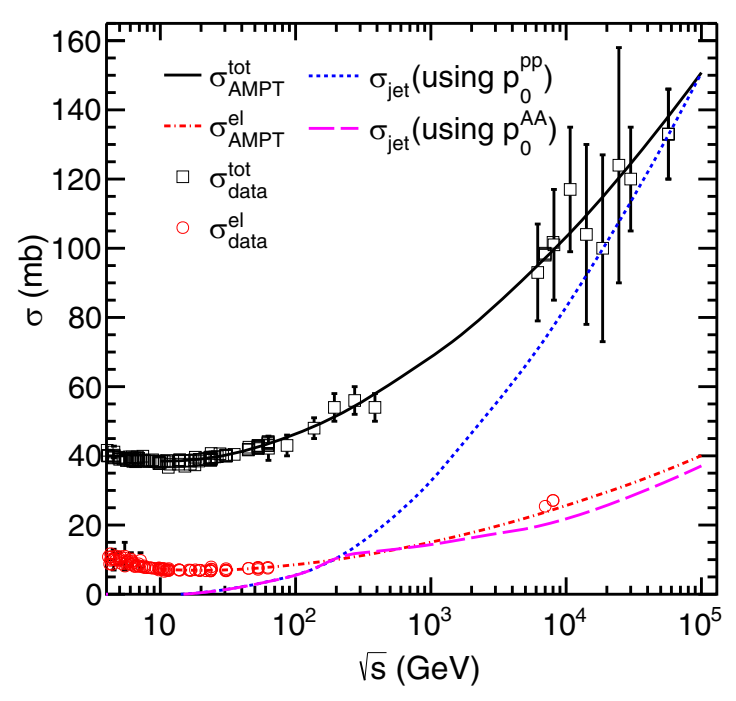

FIG. 3. Total and elastic cross sections versus the colliding energy of $p p$ collisions from the experimental data (symbols) in comparison with the AMPT results (solid and dot-dashed curves); jet cross section per $p p$ collision, $\sigma_{\text {jet }}$, is also shown for $p p$ (dotted) and $A A$ (dashed) collisions.

\section{Fitting the two-component model to $p p$ and $p \bar{p}$ cross section data}

The two parameters, $p_{0}$ and $\sigma_{\text {soft }}$, in the HIJING 1.0 model directly affect the total and inelastic cross sections of $p p$ and $p \bar{p}$ collisions, as shown in Sec. II A. In the current AMPT model that uses the Duke-Owens PDFs, constant values of $p_{0}=2.0 \mathrm{GeV} / c$ and $\sigma_{\text {soft }}=57 \mathrm{mb}$ (at high energies [27]) are found to be able to describe the experimental cross sections of $p p$ and $p \bar{p}$ collisions $[10,11]$. This is no longer the case after we use the CTEQ PDFs here, or when the GRV PDFs were used for the HIJING 2.0 model [22]. Instead, energydependent $p_{0}(s)$ and $\sigma_{\text {soft }}(s)$ values are needed.

Again we use the experimental total and inelastic cross sections of $p p$ and $p \bar{p}$ collisions within the energy range $4<\sqrt{s}<10^{5} \mathrm{GeV}$, as shown in Fig. 3, to determine these two parameters at a given energy. To fit the experimental cross sections, we minimize the sum of squared relative difference between the model results and the cross section data points. We then determine the following fit functions of $p_{0}(s)$ and $\sigma_{\text {soft }}(s)$ :

$$
\begin{aligned}
p_{0}^{p p}(s)= & -1.71+1.63 \ln (\sqrt{s})-0.256 \ln ^{2}(\sqrt{s}) \\
& +0.0167 \ln ^{3}(\sqrt{s}), \\
\sigma_{\text {soft }}(s)= & 45.1+0.718 \ln (\sqrt{s})+0.144 \ln ^{2}(\sqrt{s}) \\
& +0.0185 \ln ^{3}(\sqrt{s}) .
\end{aligned}
$$

In the above, $p_{0}^{p p}$ and $\sigma_{\text {soft }}$ are in the unit of $\mathrm{GeV} / c$ and $\mathrm{mb}$, respectively; while the center-of-mass colliding energy $\sqrt{s}$ is in the unit of GeV. Note that we have denoted the above minijet transverse momentum cutoff as $p_{0}^{p p}$ because it represents the $p_{0}$ fit function for $p p$ collisions, while we shall see in Sec. III B that $p_{0}$ needs to be $A$-dependent to reproduce the particle yields in $A A$ collisions at very high energies such as LHC energies. Also, $p_{0}$ values are only relevant when the 


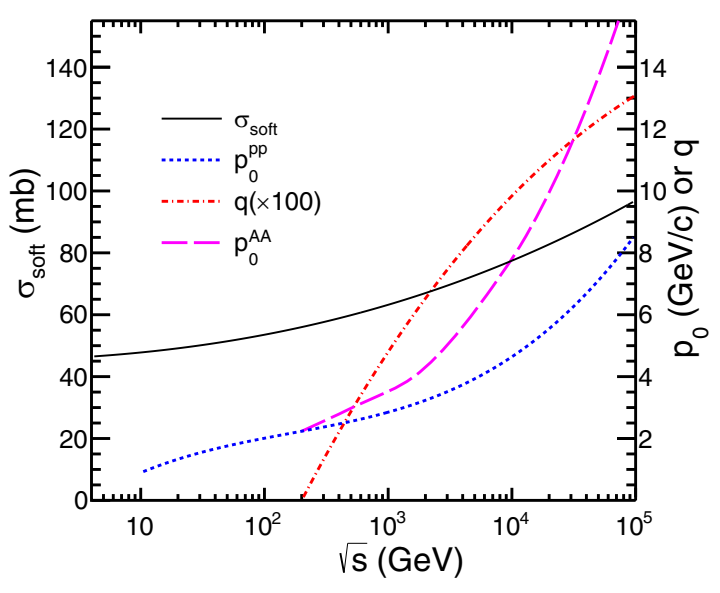

FIG. 4. Fitted $\sigma_{\text {soft }}$ function (solid curve), $p_{0}^{p p}$ function for $p p$ collisions (dotted), and the $q$ function (dot-dashed, scaled up by a factor of 100) versus the colliding energy. Dashed curve represents the $p_{0}^{A A}$ function for central $A A$ collisions after the nuclear scaling of $p_{0}$ of Eq. (10). Note that $p_{0}$ is only relevant at $\sqrt{s_{\mathrm{NN}}}>10 \mathrm{GeV}$.

center-of-mass energy per nucleon-pair is higher than $10 \mathrm{GeV}$, because the jet production in the HIJING model is switched off at $\sqrt{s}<10 \mathrm{GeV}$.

Figure 4 shows these two fit functions versus the colliding energy. We see that both show a strong energy dependence, especially the minijet cutoff scale $p_{0}$. Because the CTEQ parametrization has much higher gluon densities at small $x$ than the Duke-Owens PDFs, it has a larger jet cross section at high colliding energies, therefore a higher $p_{0}$ value than the previous value of $2 \mathrm{GeV} / c$ is needed to reproduce the total and elastic cross section data at high energies. As shown in Fig. 3, the above fit functions of $p_{0}(s)$ and $\sigma_{\text {soft }}(s)$ allow the updated AMPT model to describe the experimental data on the total and elastic cross sections of $p p$ collisions within a wide energy range $4<\sqrt{s}<10^{5} \mathrm{GeV}$.

\section{RESULTS ON PARTICLE PRODUCTIONS}

We now study particle productions in $p p$ and $A A$ collisions with the string melting version of the updated AMPT model and compare with the experimental data. In the string melting AMPT model [4,5], the initial partons are produced through the intermediate step of Lund string fragmentation, where hadrons and resonances from the fragmentation process are decomposed into (anti)quarks according to the quark model. Therefore, the initial phase-space distribution of the produced partons depends on the string fragmentation parameters, particularly the $a$ and $b$ parameters in the Lund symmetric fragmentation function:

$$
f(z) \propto z^{-1}(1-z)^{a} \exp \left(-b m_{\mathrm{T}}^{2} / z\right) .
$$

In the above, $z$ is the light-cone momentum fraction of the produced hadron with respect to the fragmenting string, and $m_{\mathrm{T}}$ is the hadron transverse mass. As a result, the final spectrum of produced particles in the AMPT model depends on the Lund $a$ and $b$ parameters [4,28]. In particular, a smaller Lund $b$ value leads to a harder $p_{\mathrm{T}}$ spectrum [28]. Note that

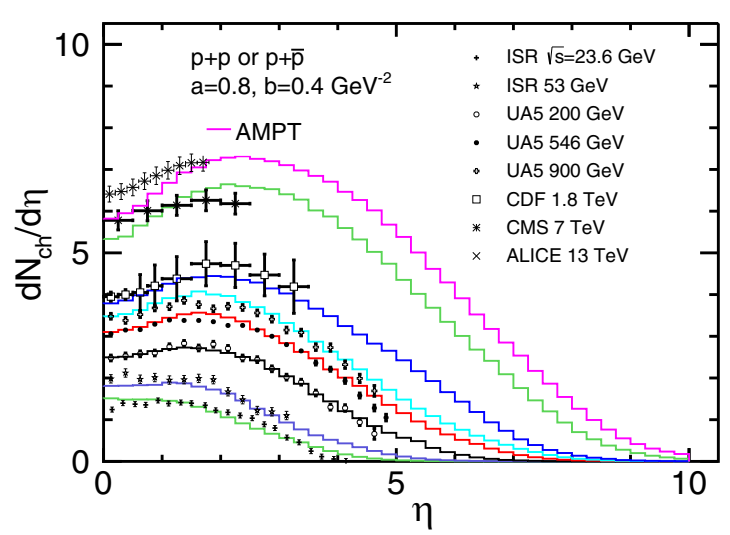

FIG. 5. Pseudorapidity distributions of charged particles in NSD $p p$ collisions at $\sqrt{s}=7 \mathrm{TeV}$ [29], NSD $p \bar{p}$ collisions at 200, 546, 900 [30], and $1800 \mathrm{GeV}$ [31], and inelastic $p p$ collisions at $23.6 \mathrm{GeV}, 53 \mathrm{GeV}$ [32], and $13 \mathrm{TeV}$ [33] from AMPT (curves) in comparison with the experimental data.

the updated AMPT model used for this study also includes the new quark coalescence [7], which respects the net-baryon conservation in each event but does not force the numbers of mesons, baryons, and antibaryons in an event to be separately conserved through the quark coalescence process. In this study, the $r_{\mathrm{BM}}$ parameter in the new coalescence, which controls the relative probability of a quark forming a baryon instead of forming a meson, is set to 0.53 for $u / d / s$ quarks. In addition, the parton cross section in ZPC is set to $3 \mathrm{mb}$.

In this section, we first investigate particle productions in $p p$ collisions at RHIC and LHC energies to determine the values of the Lund $a$ and $b$ parameters. We then apply the same Lund $a$ and $b$ values as well as the same minijet cutoff value $p_{0}$ to $A A$ collisions, and we shall see that they fail to describe the experimental data of central $A A$ collisions. We then keep the same Lund $a$ value but determine the Lund $b$ value and the $A$-scaled $p_{0}$ value that are needed for the string melting AMPT model to reproduce the overall particle productions in central $A A$ collisions at RHIC and LHC energies.

\section{A. Particle productions in $p p$ collisions}

With the $p_{0}^{p p}(s)$ minijet cutoff function, using constant Lund fragmentation parameters of $a=0.8$ and $b=$ $0.4 \mathrm{GeV}^{-2}$ allows the string melting AMPT model to reasonably describe the $p p$ and $p \bar{p}$ data in both the $d N_{c h} / d \eta$ distributions and the $p_{\mathrm{T}}$ spectra. Figure 5 shows charged particle pseudo-rapidity distributions from the updated AMPT model in comparison with the experimental data of $p p$ and $p \bar{p}$ collisions from $\sim 20 \mathrm{GeV}$ to $13 \mathrm{TeV}$. Note that we use the same procedure to select events for the AMPT analysis as that used for the experimental data. For example, NSD events in the UA5 data refer to events that contains at least one hit simultaneously on both sides of the chambers covering $2<$ $|\eta|<5.6$, while for the CDF and CMS data they refer to the ranges of $3.2<|\eta|<5.9$ and $2.9<|\eta|<5.2$, respectively.

Figure 6 shows the transverse momentum spectra of charged particles in $p p$ and $p \bar{p}$ collisions from the string melting AMPT model at different colliding energies in 


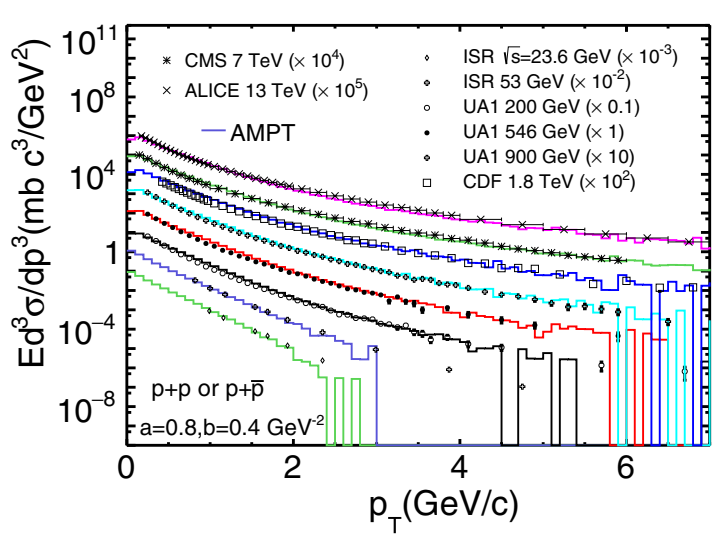

FIG. 6. Invariant cross sections of charged particles versus $p_{\mathrm{T}}$ in $p p$ collisions at $\sqrt{s}=23.6$ and $53 \mathrm{GeV}$ [32], $p \bar{p}$ collisions at 200, 546, 900 [34], and $1800 \mathrm{GeV}$ [35], and $p p$ collisions at 7 [36] and $13 \mathrm{TeV}$ [37] from the AMPT model in comparison with the experimental data.

comparison with data. Note that for $\sqrt{s}=7$ and $13 \mathrm{TeV}$, we have converted the data on $E d^{3} N / d p^{3}$ to $E d^{3} \sigma / d p^{3}$. We have used the same $\eta$ range in calculating the AMPT results as that in the experimental data: $|\eta|<0.35$ for $\sqrt{s}=23.6$ and $53 \mathrm{GeV},|\eta|<2.5$ for 200,546 , and $900 \mathrm{GeV},|\eta|<1$ for 1.8 $\mathrm{TeV},|\eta|<0.8$ for $7 \mathrm{TeV}$, and $|\eta|<2.4$ for $13 \mathrm{TeV}$.

For identified particles, we compare in the upper panel of Fig. 7 the string melting AMPT results on $d N / d y$ at midrapidity for pions, kaons, protons, and antiprotons in $p p$ collisions as functions of the colliding energy from $6 \mathrm{GeV}$ to $13 \mathrm{TeV}$. The experimental data are shown by symbols for comparison. We see that the string melting AMPT model can reasonably describe the energy dependence of most of these hadrons, including the fast increase of the antiproton yields with the colliding energy and the nonmonotonous energy dependence of the proton $d N / d y$.

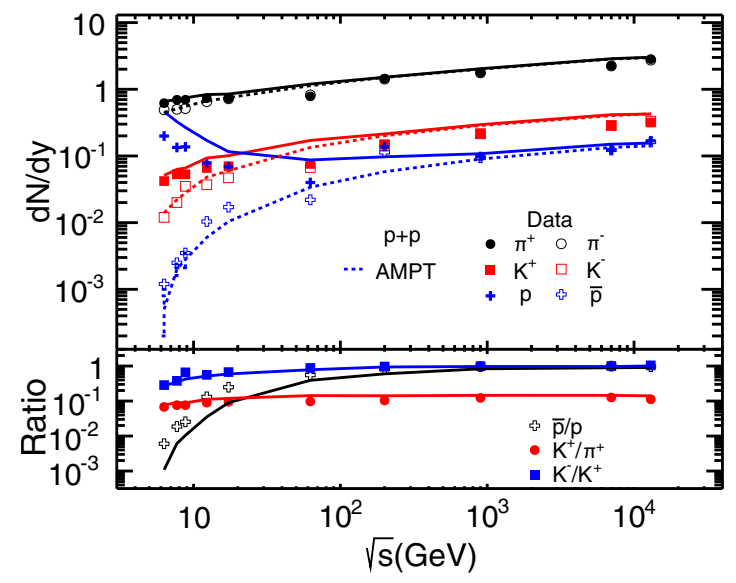

FIG. 7. Identified particle $d N / d y$ and particle ratios at midrapidity in $p p$ collisions versus the colliding energy; curves represent the AMPT results with $a=0.8$ and $b=0.4 \mathrm{GeV}^{-2}$, while symbols represent the experimental data from NA61SHINE [38], PHENIX [39], STAR [40], and ALICE [41-43].
We also see from Fig. 7 that charged pion and kaon productions from the AMPT model show good consistency with the $p p$ experimental data at different colliding energies, including the $K^{+} / \pi^{+}$and $K^{-} / K^{+}$ratios as functions of the colliding energy as shown in the lower panel of Fig. 7. However, the AMPT model here underestimates the antiproton yield and overestimates the proton yield at lower colliding energies. As a result, the $\bar{p} / p$ ratios from the AMPT model are lower than the data at the lower RHIC energies. Note that in Fig. 7 the PHENIX proton and antiproton data [39] shown at $62.4 \mathrm{GeV}$ are corrected for feed-down effects, but the STAR proton and antiproton data [40] shown at $200 \mathrm{GeV}$ are not.

\section{B. Particle productions in AA collisions}

Now we investigate results from the updated AMPT model on particles productions in nucleus-nucleus collisions. First we take the same parameters as for $p p$ collisions, i.e., Lund fragmentation parameters $a=0.8, b=0.4 \mathrm{GeV}^{-2}$, and the $p_{0}^{p p}(s)$ minijet cutoff function. Figure 8 shows the $d N / d y$ (left panels) and $p_{\mathrm{T}}$ spectra (right panels) of $\pi^{+}, K^{+}, p$, and $\bar{p}$ for $0-5 \%$ central $\mathrm{Au}+\mathrm{Au}$ collisions at $\sqrt{s_{\mathrm{NN}}}=200 \mathrm{GeV}$ and $0-5 \%$ central $\mathrm{Pb}+\mathrm{Pb}$ collisions at $2.76 \mathrm{TeV}$, where results from the updated AMPT model are being compared to the experimental data [44-46]. Note that we show the PHENIX proton and antiproton data because they have been corrected for feed-down effects. Also, the kaon and (anti)proton $d N / d y$ values from both the model and the experimental data have been multiplied by a constant factor for easier identification.

We see from Fig. 8 that the updated AMPT model with $a=0.8, b=0.4 \mathrm{GeV}^{-2}$, and the $p_{0}^{p p}(s)$ minijet cutoff significantly overestimates the yields of most of these particles for central heavy ion collisions at both RHIC and LHC energies. Also, the $p_{\mathrm{T}}$ spectra of these particles from the AMPT model are mostly softer than the data for both collision systems. Moreover, with the $p_{0}^{p p}$ minijet cutoff and EPS09sNLO nuclear shadowing, we find it impossible to reproduce the overall particle yields of $\mathrm{Pb}+\mathrm{Pb}$ collisions at LHC energies regardless of the Lund $a$ and $b$ values.

We thus introduce the following $A$-scaling of $p_{0}$, which increases the minijet cutoff $p_{0}$ for central AA collisions at high energies such as the LHC:

$$
\begin{aligned}
p_{0}^{A A}= & p_{0}^{p p} A^{q(s)}, \\
q(s)= & 0.0334 \ln \left(\frac{\sqrt{s}}{200}\right)-0.00232 \ln ^{2}\left(\frac{\sqrt{s}}{200}\right) \\
& +0.0000541 \ln ^{3}\left(\frac{\sqrt{s}}{200}\right), \quad \text { for } \sqrt{s} \geqslant 200 \mathrm{GeV} .
\end{aligned}
$$

In the above, $\sqrt{s}$ refers to $\sqrt{s_{\mathrm{NN}}}$ in $A A$ collisions and is in the unit of GeV. This $q(s)$ fit function is shown in Fig. 4, where it is zero at $\sqrt{s_{\mathrm{NN}}} \leqslant 200 \mathrm{GeV}$, reaches a value of 0.13 at $\sqrt{s_{\mathrm{NN}}}=10^{5} \mathrm{GeV}$, and approaches 0.16 at $\sqrt{s_{\mathrm{NN}}} \sim 10^{7} \mathrm{GeV}$. The nuclear-scaled $p_{0}$ function, $p_{0}^{A A}$, is also shown in Fig. 4, where we can see the significant increase of the cutoff scale $p_{0}$ in central $A A$ collisions at LHC energies and beyond. The above nuclear scaling of the minijet momentum cutoff scale $p_{0}$ is motivated by the physics of color glass condensate [47], 

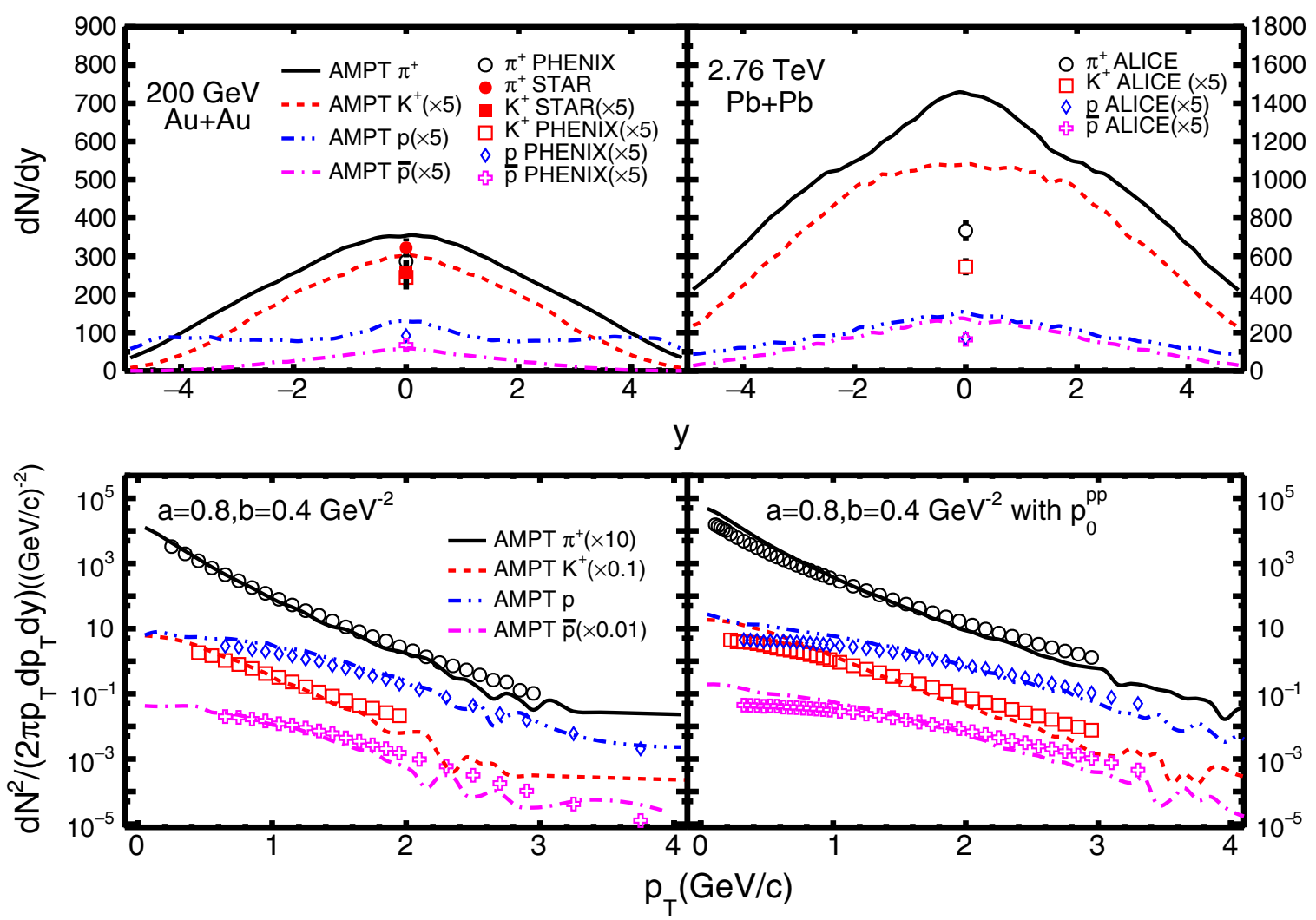

FIG. 8. Identified particle $d N / d y$ distributions (upper panels) and $p_{\mathrm{T}}$ spectra (lower panels) for $0-5 \%$ central $\mathrm{Au}+\mathrm{Au}$ collisions at $200 \mathrm{GeV}$ (left panels) and $0-5 \%$ central $\mathrm{Pb}+\mathrm{Pb}$ collisions at $2.76 \mathrm{TeV}$ (right panels). Curves represent the AMPT results using the same Lund fragmentation parameters and $p_{0}$ as for $p p$ collisions, while symbols represent experimental data $[44,46]$.

where the saturation momentum scale $Q_{s}$ depends on the nuclear size as $Q_{s} \propto A^{1 / 6}$ in the saturation regime for small- $x$ gluons in $A A$ collisions at high-enough energies.

To obtain the above $q(s)$ fit function, first we decide to keep using the EPS09s nuclear shadowing, although it has significant uncertainties on its gluon shadowing function at small $x$ [13]. We also use the same Lund $a$ value of 0.8 for $A A$ collisions as for $p p$ collisions, unlike in studies with the previous AMPT model [4,7]. In addition, we find that a significantly smaller value for the Lund $b$ parameter, $b=0.15 \mathrm{GeV}^{-2}$, is needed to describe particle productions in $A A$ collisions. This was also the case for the previous string melting version of the AMPT model [6,28]. Note that throughout this study we use the default PYTHIA value of 0.30 for the relative production of strange to nonstrange quarks, instead of imposing an upper limit of 0.40 as done for the string melting version of the previous AMPT model [28]. We then use the overall particle yields of central $\mathrm{Au}+\mathrm{Au}$ collisions at the higher RHIC energies and central $\mathrm{Pb}+\mathrm{Pb}$ collisions at LHC energies to determine the corresponding $q$ value at each energy, and finally a fit of the obtained $q$ values is done to arrive at the above $q(s)$ fit function.

Figure 9 shows the $d N / d y$ distributions (left panels) and $p_{\mathrm{T}}$ spectra (right panels) from the AMPT model using the new Lund $b$ parameter and $p_{0}^{A A}(s)$ cutoff in comparison with the experimental data. We see that most of the $d N / d y$ data of $\pi^{+}, K^{+}, p$, and $\bar{p}$ in these central heavy ion collisions can now be reasonably reproduced. The $p_{\mathrm{T}}$ spectra are also much harder than those in Fig. 8 and mostly consistent with the corresponding heavy ion data, due to the smaller value of the Lund $b$ parameter [28].

In Fig. 10, the energy dependencies of identified particle yields at midrapidity are shown in the upper panel for $0-5 \%$ central $\mathrm{Au}+\mathrm{Au}$ collisions at RHIC energies and 0-5\% central $\mathrm{Pb}+\mathrm{Pb}$ collisions at LHC energies. The corresponding particle ratios are shown in the lower panel. Note that the rapidity range at $2.76 \mathrm{TeV}$ is $|y|<0.5$ while at other energies is $|y|<0.1$, and that the PHENIX (anti)protons data at 62.4 and $130 \mathrm{GeV}$ are not corrected for feed-down from weak decays. We see from Fig. 10 that the yields of charged pions and kaons as well as their ratios are well reproduced by the updated AMPT model. However, similar to the trend in $p p$ collisions, at lower energies the string melting AMPT model underestimates the antiproton yield but tends to overestimates the proton yield at midrapidity. As a result, the midrapidity $\bar{p} / p$ ratios from the string melting AMPT model at the lower RHIC energies are significantly smaller than the experimental data. However, the AMPT model can reasonably reproduce the (anti)proton data for central $\mathrm{Pb}+\mathrm{Pb}$ collisions at the $\mathrm{LHC}$ energy of $2.76 \mathrm{TeV}$. These features are similar to those in the earlier study that used the previous string melting AMPT model with the new quark coalescence [7]. 

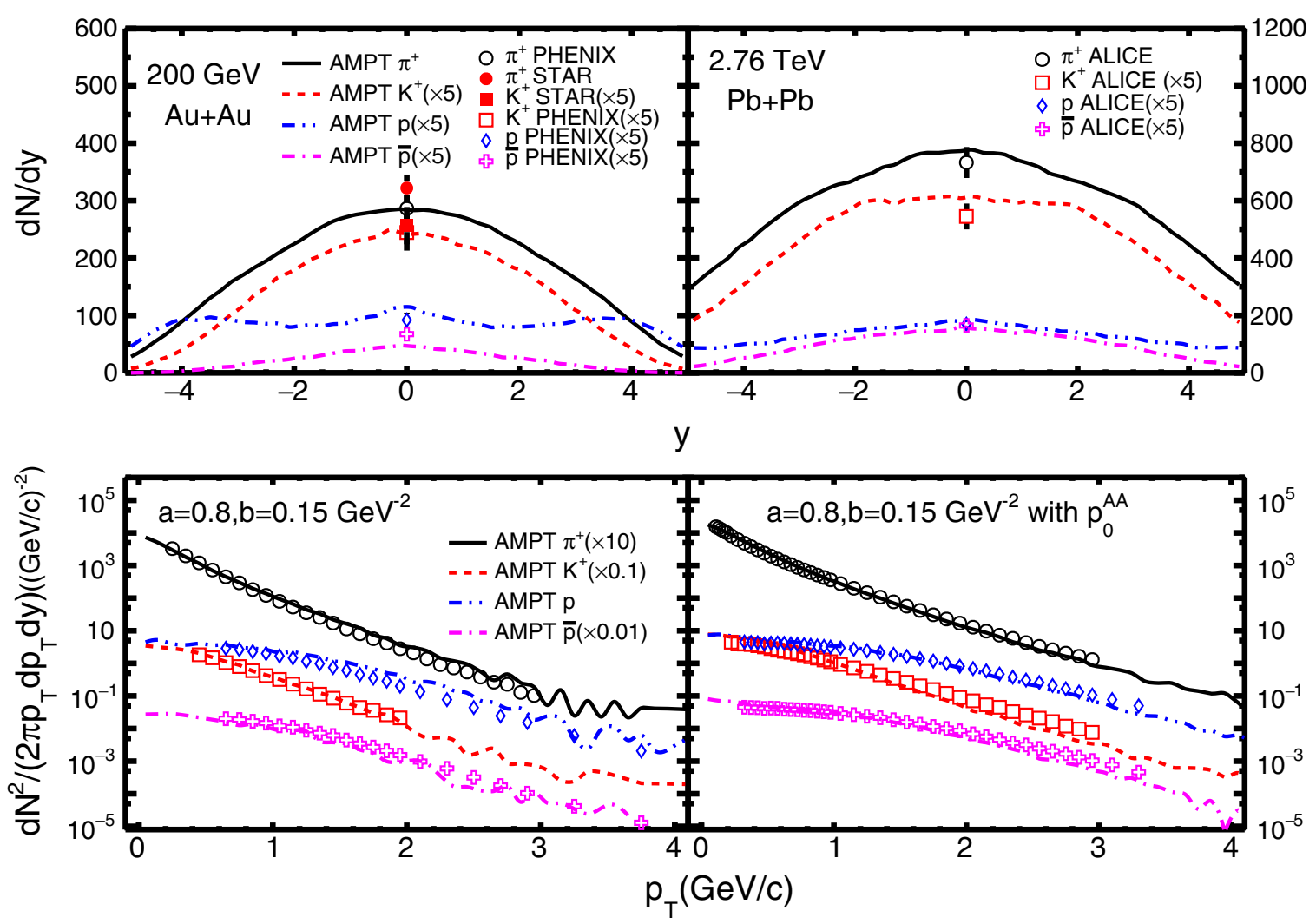

FIG. 9. Same as Fig. 8 but using Lund $b=0.15 \mathrm{GeV}^{-2}$ and $A$-scaled minijet cutoff $p_{0}^{A A}(s)$ for central $A A$ collisions; note that $p_{0}^{A A}=p_{0}^{p p}$ at $\sqrt{s_{\mathrm{NN}}}=200 \mathrm{GeV}$.

\section{DISCUSSIONS}

Since the EPS09s nuclear shadowing is impact parameterdependent and diminishes for nucleons near the edge of the nucleus, we expect the effect of nuclear shadowing to depend

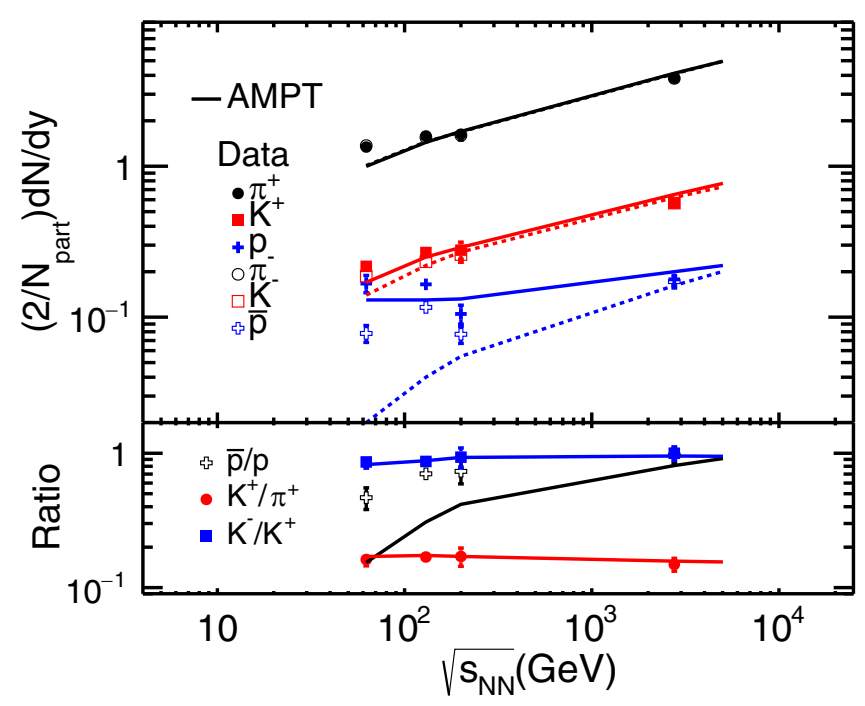

FIG. 10. Identified particle $d N / d y$ and particle ratios at midrapidity in $0-5 \%$ central $\mathrm{Au}+\mathrm{Au}$ or $\mathrm{Pb}+\mathrm{Pb}$ collisions versus the colliding energy; curves represent AMPT results, while symbols represent experimental data at $62.4 \mathrm{GeV}$ [45], $130 \mathrm{GeV}$ [48], 200 $\mathrm{GeV}$ [44], and 2.76 TeV [46]. on centrality and vanish for very peripheral $A A$ collisions. This is shown in Fig. 11 by the centrality dependence of charged particle $d N / d \eta$ within $|\eta|<0.5$ divided by $N_{\text {part }} / 2$ for $\mathrm{Au}+\mathrm{Au}$ collisions at $200 \mathrm{GeV}$ (thin curves, data from Ref. [49]) and $\mathrm{Pb}+\mathrm{Pb}$ collisions at $2.76 \mathrm{TeV}$ and $5.02 \mathrm{TeV}$ (medium and thick curves, respectively, data from Ref. [50]

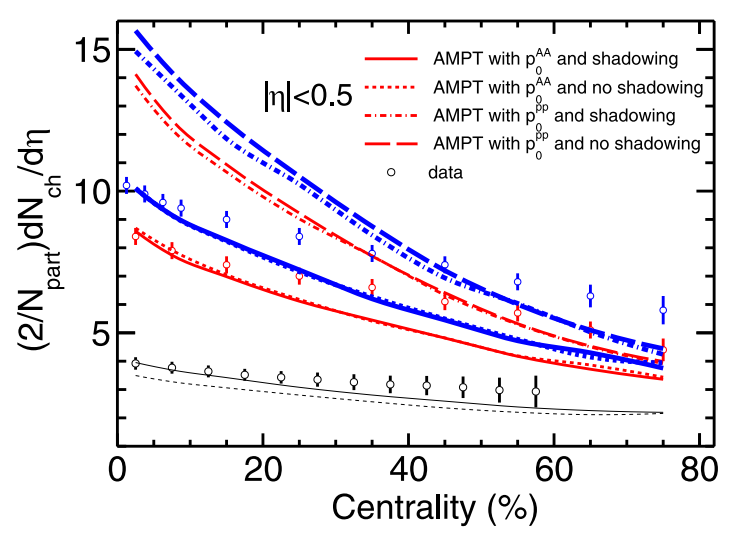

FIG. 11. Centrality dependence of midrapidity $d N_{c h} / d \eta$ divided by $N_{\text {part }} / 2$ from the AMPT model for $\mathrm{Au}+\mathrm{Au}$ collisions at $200 \mathrm{GeV}$ and $\mathrm{Pb}+\mathrm{Pb}$ collisions at $2.76 \mathrm{TeV}$ and $5.02 \mathrm{TeV}$ with (solid) and without (dotted) nuclear shadowing in comparison with data [49-51]. Also shown are AMPT results at LHC energies without using the $A$-scaling of $p_{0}$ for central $A A$ collisions with (dot-dashed) or without (dashed) nuclear shadowing, which are applicable to very peripheral $A A$ collisions. 
and Ref. [51], respectively). Note that centrality is determined according to the number of charged particles detected by the beam-beam counters that cover $3.0<|\eta|<3.9$ at $200 \mathrm{GeV}$ or by the V0 detectors that cover $2.8<\eta<5.1$ and $-3.7<\eta<1.7$ at $2.76 \mathrm{TeV}$ or $5.02 \mathrm{TeV}$. The same centrality criterion is used in the analysis of our model results, and we take $N_{\text {part }}$ as the total number of nucleon participants from both the projectile and target nuclei due to inelastic collisions in the AMPT calculations.

As expected, we see in Fig. 11 that the shadowing effect is very small for peripheral collisions. Actually, the figure shows that nuclear shadowing has a small effect on charged particle yields at all centralities for $A A$ collisions from the top RHIC energy to LHC energies. This is because of the large $p_{0}$ values at high energies as shown in Fig. 4 as well as the relatively weak EPS09sNLO nuclear shadowing at large $Q^{2}$ values as shown in Fig. 2. A large $p_{0}$ value suppresses the minijet component's contribution, which is sensitive to the nuclear shadowing, to the particle yields relative to the soft component's contribution. If the nuclear scaling of $p_{0}$ is not applied, we can see in Fig. 11 that nuclear shadowing would reduce the overall particle yields by a finite (but still small) amount for central $\mathrm{Pb}+\mathrm{Pb}$ collisions at LHC energies, because $p_{0}^{p p}$ is significantly lower than $p_{0}^{A A}$ there.

However, Fig. 11 shows that the $A$-scaling of $p_{0}$ has a large effect on charged particle yields in $A A$ collisions at LHC energies, especially for more central collisions. As mentioned earlier, the string melting AMPT model significantly overestimates the charged particle yields in central $\mathrm{Pb}+\mathrm{Pb}$ collisions at LHC when it uses $p_{0}^{p p}$, the same minijet cutoff scale as for $p p$ collisions. After the $A$-scaling of $p_{0}$, however, the minijet cutoff scale in $A A$ collisions $\left(p_{0}^{A A}\right)$ at LHC energies becomes significantly higher and thus $\sigma_{\text {jet }}$ becomes much smaller, as shown in Fig. 3 by the dashed line that is much lower than the dotted line at LHC energies. This leads to a significant decrease of the charge particle yields at LHC energies, especially for central $A A$ collisions where the binary scaling of minijet productions makes them more sensitive to the minijet cutoff $p_{0}$.

For peripheral $A A$ collisions however, we expect no need for the $A$-scaling of $p_{0}$, because participant nucleons there are near the edge of the nucleus and should be almost free of saturation effects. Since we have not implemented this impact parameter-dependent nuclear scaling of $p_{0}$ and the current $A$-scaling of Eq. (10) is only valid for central $A A$ collisions, we show in Fig. 11 the $\mathrm{LHC} \mathrm{Pb}+\mathrm{Pb}$ results without using the $A$-scaling of $p_{0}$ (dot-dashed lines and dashed lines), which are more suitable for peripheral collisions. Indeed, we see that the AMPT results without the $A$-scaling of $p_{0}$ give higher charged particle yields and are closer to the experimental data for peripheral collisions than the AMPT results with $A$-scaling of $p_{0}$. Also note that, since we have found that the Lund $b$ value is much smaller in central $A A$ collisions than in $p p$ collisions, the Lund $b$ value should depend on the system size or centrality, and increasing its value for peripheral $A A$ collisions (similar to $p p$ collisions) could further improve the description of charged particle yields there.

We have seen that the minijet cutoff scale $p_{0}$ becomes increasingly large with energy and can be more than 4 or even $6 \mathrm{GeV} / c$. However, it is questionable to treat transverse momentum exchanges below such a high value of $p_{0}$ as soft physics with the Lund string fragmentation, while the production of charm particles is usually viewed as a perturbativeQCD process where the FONNL approach has been very successful [52]. Therefore, the two-component model such as HIJING may be problematic for the initial condition at very high energies. For example, the need for us to introduce the nuclear scaling of $p_{0}$ for $A A$ collisions at LHC energies and above may indicate the importance of saturation physics for large systems at very high energies. In addition, the current parton cascade in the AMPT model only includes elastic parton scatterings [14]. However, inelastic parton interactions [53] affect the parton abundance and momentum spectrum at high energies, and these effects are expected to be energyand centrality-dependent. Therefore, including inelastic parton scatterings should improve the physics of a multiphase transport model [54].

The updated AMPT model has not shown obvious phenomenological improvements over the previous AMPT model when compared with the experimental data in this study, except that the updated model uses the same Lund $a$ value for $p p$ and $A A$ collisions at all energies and thus removes the uncertainty of this parameter present in the previous AMPT model. However, the updated AMPT model should be more robust in its physics because of its inclusion of modern parton PDFs in the nuclei. Therefore, we expect it to provide a better foundation for future model developments and also show improvements in certain observables such as heavy flavor productions [55].

\section{SUMMARY}

A multiphase transport model has been using the old DukeOwens parton distribution functions for the free proton and a schematic nuclear shadowing parametrization. This leads to significant uncertainties in its ability to address heavy flavor and/or high- $p_{\mathrm{T}}$ particles, because they are produced by perturbative-QCD processes and thus directly depend on the parton distribution functions of nuclei. In this study, we have incorporated a newer set of free proton parton distribution functions, the CTEQ6.1M set, and the impact parameterdependent EPS09sNLO nuclear shadowing in an updated AMPT model. We first determine the energy dependence of two key parameter functions, $p_{0}(s)$ and $\sigma_{\text {soft }}(s)$, in the HIJING two-component model by fitting the experimental data on total and inelastic cross sections of $p p$ and $p \bar{p}$ collisions from $\sqrt{s} \sim 4 \mathrm{GeV}$ to $13 \mathrm{TeV}$. We then compare particle productions from the string melting version of the updated AMPT model with the experimental data in both $p p$ and $A A$ collisions at RHIC and LHC energies. We find that the $p_{0}(s)$ function and the constant values for the Lund string fragmentation parameters that can reasonably describe the particle yields and $p_{\mathrm{T}}$ spectra in $p p$ collisions fail to describe central $A A$ collisions at LHC energies. Therefore, we introduce a nuclear scaling of the minijet transverse momentum cutoff $p_{0}$ for central $A A$ collisions at high energies that is motivated by the color glass condensate picture. Then the string melting AMPT 
model can also reasonably describe the overall particle yields and $p_{\mathrm{T}}$ spectra of $A A$ collisions at both RHIC and LHC energies. We expect the updated AMPT model to provide more reliable descriptions of heavy flavor and high- $p_{\mathrm{T}}$ observables in relativistic collisions of both small and large systems. It also serves as a good foundation for further improvements of the model.

\section{ACKNOWLEDGMENTS}

This work is supported by the Ministry of Science and Technology (MoST) of China under 973-Project No. 2015CB856901 (F.L. and S.S.) and the National Natural Science Foundation of China under Grants No. 11890711 and No. 11628508 (Z.W.L., F.L., and S.S.).
[1] I. Arsene et al. (BRAHMS Collaboration), Nucl. Phys. A 757, 1 (2005); B. B. Back et al. (PHOBOS Collaboration), ibid. 757, 28 (2005); J. Adams et al. (STAR Collaboration), ibid. 757, 102 (2005); K. Adcox et al. (PHENIX Collaboration), ibid. 757, 184 (2005).

[2] U. Heinz and R. Snellings, Annu. Rev. Nucl. Part. Sci. 63, 123 (2013).

[3] W. Busza, K. Rajagopal, and W. van der Schee, Annu. Rev. Nucl. Part. Sci. 68, 339 (2018).

[4] Z. W. Lin, C. M. Ko, B. A. Li, B. Zhang, and S. Pal, Phys. Rev. C 72, 064901 (2005).

[5] Z. W. Lin and C. M. Ko, Phys. Rev. C 65, 034904 (2002).

[6] G. L. Ma and Z. W. Lin, Phys. Rev. C 93, 054911 (2016).

[7] Y. He and Z. W. Lin, Phys. Rev. C 96, 014910 (2017).

[8] L. Y. Zhang, J. H. Chen, Z. W. Lin, Y. G. Ma, and S. Zhang, Phys. Rev. C 98, 034912 (2018).

[9] AMPT source codes are available at http://myweb.ecu.edu/linz/ ampt/.

[10] X. N. Wang and M. Gyulassy, Phys. Rev. D 44, 3501 (1991).

[11] M. Gyulassy and X. N. Wang, Comput. Phys. Commun. 83, 307 (1994).

[12] J. Pumplin, D. R. Stump, J. Huston, H. L. Lai, P. M. Nadolsky, and W. K. Tung, J. High Energy Phys. 07 (2002) 012.

[13] I. Helenius, K. J. Eskola, H. Honkanen, and C. A. Salgado, J. High Energy Phys. 07 (2012) 073.

[14] B. Zhang, Comput. Phys. Commun. 109, 193 (1998).

[15] B. A. Li and C. M. Ko, Phys. Rev. C 52, 2037 (1995).

[16] E. Eichten, I. Hinchliffe, K. D. Lane, and C. Quigg, Rev. Mod. Phys. 56, 579 (1984); 58, 1065 (1986).

[17] T. K. Gaisser and F. Halzen, Phys. Rev. Lett. 54, 1754 (1985).

[18] G. Pancheri and Y. N. Srivastava, Phys. Lett. B 182, 199 (1986).

[19] D. W. Duke and J. F. Owens, Phys. Rev. D 30, 49 (1984).

[20] Z. W. Lin, Indian J. Phys. 85, 837 (2011).

[21] M. Gluck, E. Reya, and A. Vogt, Z. Phys. C 67, 433 (1995).

[22] W. T. Deng, X. N. Wang, and R. Xu, Phys. Rev. C 83, 014915 (2011).

[23] A. Accardi, L. T. Brady, W. Melnitchouk, J. F. Owens and N. Sato, Phys. Rev. D 93, 114017 (2016).

[24] K. J. Eskola, H. Paukkunen, and C. A. Salgado, J. High Energy Phys. 04 (2009) 065.

[25] K. J. Eskola, H. Paukkunen, and C. A. Salgado, J. High Energy Phys. 07 (2008) 102.

[26] D. Stump, J. Huston, J. Pumplin, W. K. Tung, H. L. Lai, S. Kuhlmann, and J. F. Owens, J. High Energy Phys. 10 (2003) 046.

[27] X. N. Wang, Phys. Rev. D 43, 104 (1991).

[28] Z. W. Lin, Phys. Rev. C 90, 014904 (2014).

[29] V. Khachatryan et al. (CMS Collaboration), Phys. Rev. Lett. 105, 022002 (2010).
[30] G. J. Alner et al. (UA5 Collaboration), Z. Phys. C 33, 1 (1986).

[31] F. Abe et al. (CDF Collaboration), Phys. Rev. D 41, 2330 (1990).

[32] W. Thome et al. (Aachen-CERN-Heidelberg-Munich Collaboration), Nucl. Phys. B 129, 365 (1977).

[33] V. Khachatryan et al. (CMS Collaboration), Phys. Lett. B 751, 143 (2015).

[34] C. Albajar et al. (UA1 Collaboration), Nucl. Phys. B 335, 261 (1990).

[35] F. Abe et al. (CDF Collaboration), Phys. Rev. Lett. 61, 1819 (1988).

[36] B. B. Abelev et al. (ALICE Collaboration), Eur. Phys. J. C 73, 2662 (2013).

[37] J. Adam et al. (ALICE Collaboration), Phys. Lett. B 753, 319 (2016).

[38] A. Aduszkiewicz et al. (NA61/SHINE Collaboration), Eur. Phys. J. C 77, 671 (2017).

[39] A. Adare et al. (PHENIX Collaboration), Phys. Rev. C 83, 064903 (2011).

[40] J. Adams et al. (STAR Collaboration), Phys. Lett. B 616, 8 (2005).

[41] K. Aamodt et al. (ALICE Collaboration), Eur. Phys. J. C 71, 1655 (2011).

[42] J. Adam et al. (ALICE Collaboration), Eur. Phys. J. C 75, 226 (2015).

[43] A. M. Sirunyan et al. (CMS Collaboration), Phys. Lett. B 779, 358 (2018)

[44] S. S. Adler et al. (PHENIX Collaboration), Phys. Rev. C 69, 034909 (2004).

[45] B. I. Abelev et al. (STAR Collaboration), Phys. Rev. C 79, 034909 (2009).

[46] B. Abelev et al. (ALICE Collaboration), Phys. Rev. C 88, 044910 (2013).

[47] L. D. McLerran and R. Venugopalan, Phys. Rev. D 49, 2233 (1994).

[48] K. Adcox et al. (PHENIX Collaboration), Phys. Rev. Lett. 88, 242301 (2002).

[49] A. Adare et al. (PHENIX Collaboration), Phys. Rev. C 93, 024901 (2016).

[50] K. Aamodt et al. (ALICE Collaboration), Phys. Rev. Lett. 106, 032301 (2011).

[51] J. Adam et al. (ALICE Collaboration), Phys. Lett. B 772, 567 (2017).

[52] M. Cacciari, S. Frixione, N. Houdeau, M. L. Mangano, P. Nason, and G. Ridolfi, J. High Energy Phys. 10 (2012) 137.

[53] Z. Xu and C. Greiner, Phys. Rev. C 71, 064901 (2005).

[54] Z. W. Lin, Acta Phys. Polon. Supp. 7, 191 (2014).

[55] L. Zheng, C. Zhang, S. Shi, and Z. W. Lin (unpublished). 